\title{
Antibacterial Properties of Nanosilver PLLA Fibrous Membranes
}

\author{
Lin Li, ${ }^{1}$ Yi Li, ${ }^{1}$ Jiashen Li, ${ }^{1}$ Lei Yao, ${ }^{1}$ Arthur F. T. Mak, ${ }^{2}$ Frank Ko, ${ }^{3}$ and Ling Qin ${ }^{4}$ \\ ${ }^{1}$ Institute of Textiles and Clothing, The Hong Kong Polytechnic University, Kowloon, Hong Kong \\ ${ }^{2}$ Department of Health Technology and Informatics, The Hong Kong Polytechnic University, Kowloon, Hong Kong \\ ${ }^{3}$ Department of Materials Engineering, AMPEL, The University of British Columbia, Vancouver, BC, Canada \\ ${ }^{4}$ Department of Orthopaedics and Traumatology, The Chinese University of Hong Kong, Shatin, Hong Kong
}

Correspondence should be addressed to Yi Li, tcliyi@inet.polyu.edu.hk

Received 15 October 2008; Revised 13 March 2009; Accepted 24 April 2009

Recommended by Alan K. T. Lau

Nanosilver has been studied as a valuable material for it strong antibacterial effects. In this study, we investigated the antibacterial properties of nano silver Poly-L-Lactic acid (Ag/PLLA) composite fibrous membranes. Ag/PLLA fibrous membranes were prepared with silver nanoparticles having weight ratio of silver nanoparticles to PLLA at 5\% (w/w). In vitro antibacterial tests were performed using Escherichia coli (E. coli) and Staphylococcus aureus (Staph.) to determine the antibacterial capability of the Ag/PLLA fibrous membranes. As the results suggested, Ag/PLLA fibrous membranes showed strong antibacterial properties. Thus, Ag/PLLA fibrous membrane can be used as an antibacterial scaffold for tissue engineering.

Copyright (C) 2009 Lin Li et al. This is an open access article distributed under the Creative Commons Attribution License, which permits unrestricted use, distribution, and reproduction in any medium, provided the original work is properly cited.

\section{Introduction}

Tissue engineering scaffolds which are supposed to be used for medical applications have applied various polymers such as degradable Poly-L-Lactide (PLLA) and Polyglycolic acid (PGA), or natural biomaterials like chitin, silk, protein, and collagen [1-13]. These biomaterials offer a promising new approach to help to restore and reconstruct defected tissues. An optimal scaffold for medical applications requires the following merits: suitable for cell adhesion and proliferation; highly porous for transport of nutrients and metabolic waste; biodegradable and bioresorbable. PLLA is a biocompatible, biodegradable, and immunologically inert synthetic polymer. Thus, PLLA was chosen in this study for designing a composition of tissue engineering scaffold.

In addition, the risk of infection requires tissue engineering scaffolds to have antibacterial properties [14]. For example, during the treatment of wound, infection is a common risk that need to be prevented for good healing of wound. Due to their strong antibacterial properties and low toxicity, silver and its compounds have been studied for many years not only for their low toxicity, but also for their antibacterial activity [14-27]. Yeo et al. found that fibers having silver showed excellent antibacterial property [28]. Electrospinning technique was employed to produce PLLA fibers to provide a matrix with wide interface for combining antibacterial materials.

Meanwhile, the nanotechnology has provided a most promising field for the new attempts of biomedical materials [14]. Such as silver nanoparticles provide larger interface to the environment than that of microscale silver particles. Li et al. (2005) reported that nanosilver had strong antibacterial efficacy with very small amount against bacteria [17]. So, nanosized silver is a good material to reduce the quantity without decreasing antibacterial activity of silver. Thus, in this study, we used the silver nanoparticles to combine with PLLA by using electrospinning technique to fabricate biodegradable tissue engineering scaffolds with antibacterial property. E. coli and Staphylococcues which often infect wound skin and other tissues were applied for antibacterial tests to investigate the antibacterial activity of Ag/PLLA fibrous membranes [28].

\section{Materials and Methods}

2.1. Materials. Poly-L-Lactide (PLLA) with molecular weight of 1000000 was purchased from Purac, Netherlands. Silver nanoparticles $(0-100 \mathrm{~nm})$ with $99.9 \%$ purity and $35 \mathrm{~nm}$ average particle size (Figure 1) were purchased from the Shenzhen Junye Nano Material Co. Ltd. 


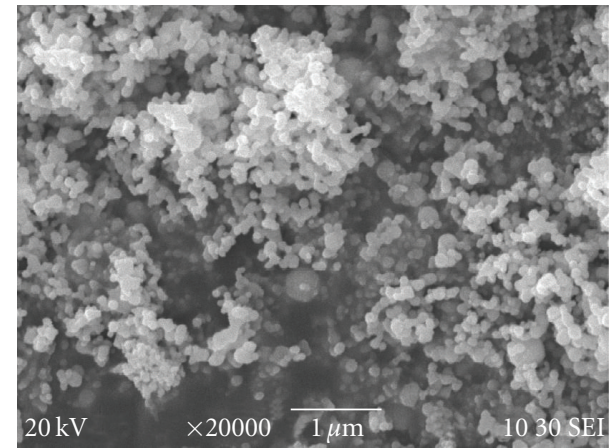

FIgure 1: SEM images of silver nanoparticle $(20 \mathrm{kV})$. Silver nanoparticles are grey and white color with the average particle size of $35 \mathrm{~nm}$.

2.2. Electrospinning of PLLA and Ag/PLLA Membranes. Electrospinning method is according to our previous study [29]. PLLA was dissolved at the weight ratio of $1 \%$ in an organic solvent mixed with the composition of chloroform and $\mathrm{N}, \mathrm{N}$-dimethlformamide (DMF) $(10: 1, \mathrm{w} / \mathrm{w})$. Silver nanoparticles were then added to the solution and stirred evenly to prepare $5 \% \mathrm{Ag} / \mathrm{PLLA}$ solution for electrospinning. PLLA and Ag/PLLA solutions were taken in a syringe with blunt needle, and fed by syringe plunger at a feeding rate of $0.3 \mathrm{~mL} / \mathrm{min}$. A grounded metal rotating drum wrapped with aluminum foil was located at a fixed distance of $10 \mathrm{~cm}$ away from the nozzle of the needle for collecting fibers. A high electric field was applied to the droplet which was coming out from the nozzle. This leads to the droplet deformation of continuous fibers. PLLA and Ag/PLLA fibrous membranes were produced under high voltage of $13 \mathrm{kV}$.

2.3. Antibacterial Test. Antibacterial test method was referred to AATCC147-2004 in this study. Escherichia coli (E. coli, ATCC25922) and Staphylococcus aureus (Staph., ATCC25923) were used for this test as the common microorganisms involved in hospital-acquired infections [17]. The test repeated three times for every specimen.

2.3.1. Procedure. The strains were cultured overnight in $10 \mathrm{~mL}$ nutrient broth to achieve the turbidity of $10^{8}$ colony forming units $(\mathrm{CFU}) / \mathrm{mL}$. Sterilized nutrient agar was dispensed by pouring $15 \pm 2 \mathrm{~mL}$ into each standard $(15 \times$ $100 \mathrm{~mm}$ ) flat bottom petri dish to obtain firmly solid agar before inoculating. Using a $4 \mathrm{~mm}$ inoculating loop, loaded one loopful of the 10 times diluted inoculums and transfer to the surface of the sterile agar by making five streaks coving the central area of a petri dish. Gently press the specimen of Ag/PLLA membrane to contact intimately with the agar surface. Then incubated at $37^{\circ} \mathrm{C}$ for 24 hours. PLLA membrane was used as a negative control. All specimens were prepared with the diameter of $5 \mathrm{~mm}$ for antibacterial test.

2.3.2. Evaluation. Examine the incubated plates for interruption of growth along the streaks of inoculum beneath the specimen and for a clear zone of inhibition beyond its edge.

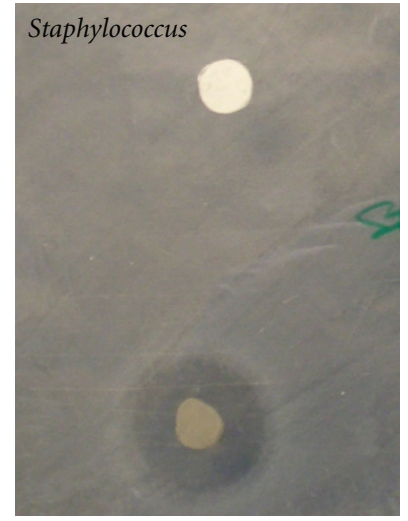

(a)

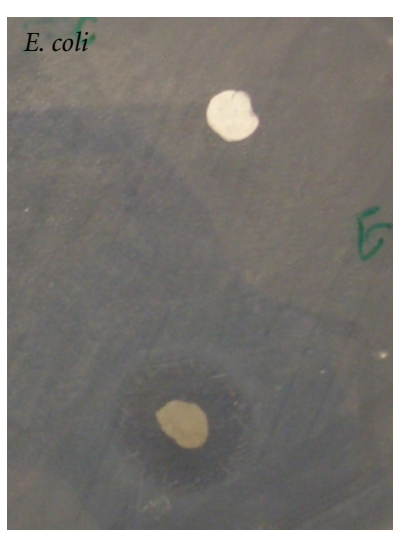

(b)
Figure 2: Antibacterial test results of Ag/PLLA using Staphylococcus (a) and E. coli (b) were used. The bacteria inhibition clear zones of both E. coli and Staph. can be observed around the Ag/PLLA membranes (lower specimen) with the average width of $5 \mathrm{~mm}$ after 24 hours incubation.

The average width of a zone of inhibition along a streak on either side of the test specimen was calculated using the following equation:

$$
W=\frac{(T-D)}{2},
$$

where $W=$ width of clear zone of inhibition in $\mathrm{mm}$; $T=$ total diameter of test specimen and clear zone in $\mathrm{mm} ; D=$ diameter of the test specimen in $\mathrm{mm}$.

2.4. Scanning Electron Microscopy (SEM). PLLA and $\mathrm{Ag} / \mathrm{PLLA}$ fibrous membranes with bacteria were sputter coated with gold, then observed by scanning electron microscopy (LEICA, Stereoscan 440, $20 \mathrm{kV}$ ).

2.5. Statistical Analysis. Values were expressed as means \pm standard deviations. Statistical differences were determined by one-way ANOVA. $P$ values of less than .05 were considered to be statistically significant.

\section{Results}

3.1. Antibacterial Activities of Ag/PLLA Fibrous Membranes. E. coli and Staph. were used for this test to evaluate the antibacterial capability of Ag/PLLA fibrous membranes. The bacteria were cultured overnight in $10 \mathrm{~mL}$ nutrient broth to achieve the turbidity of $10^{8}$ colony forming units $(\mathrm{CFU}) / \mathrm{mL}$. A loop of each kind of bacteria at the concentration of $10^{8} \mathrm{CFU} / \mathrm{mL}$ was taken to evenly streak the agar filled petri dishes. Each of the circular specimens with $5 \mathrm{~mm}$ diameter was gently pressed on the inoculated agar surface before incubation at $37^{\circ} \mathrm{C}$ for 24 hours.

As shown in Figure 2, the upper specimens were PLLA membranes, the lower specimens were Ag/PLLA membranes. All the membranes were freshly prepared. After 24 hours incubation, obvious bacteria inhibition clear zones of 


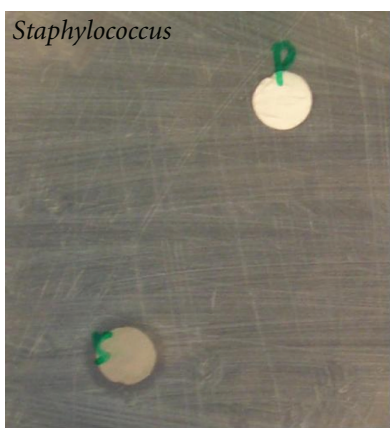

(a)

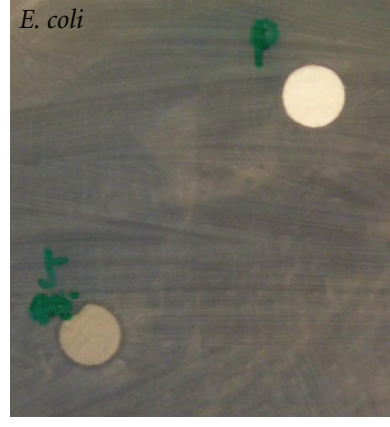

(b)
Figure 3: Antibacterial activity of Ag/PLLA fibrous membrane (lower specimen) reduced after exposure in the air for 8 weeks. The average width of E. coli and Staph. inhibition clear zones decreased to $1.2 \mathrm{~mm}$ and $1.5 \mathrm{~mm}$, respectively, after 24 hours incubation.

Ag/PLLA membranes could be seen, the average width of the zones was $55.0 \pm 0.1 \mathrm{~mm}$. While no clear zone of inhibition was seen around PLLA membranes. Both Staph. and E. coli had the same results which indicated the strong antibacterial activity of Ag/PLLA.

After exposure in the air for 8 weeks, Ag/PLLA membranes were still observed antibacterial activity. The average width of bacterial inhibition zones of Ag/PLLA membranes was $1.2 \pm 0.1 \mathrm{~mm}$ with E. coli, and $1.5 \pm 0.1 \mathrm{~mm}$ with Staph. According to Table 1, the antibacterial activity was found to decrease significantly (see Figure 3 ).

These results showed that Ag/PLLA membranes had strong antibacterial capability. Although the membranes were exposure in the air for a long term, they were still antibacterial.

3.2. SEM Observation of Staph. on PLLA and Ag/PLLA Fibrous Membranes. The morphological changes of bacteria were also observed through SEM using Staph. $20 \mu \mathrm{L}$ diluted inoculum of Staph. at the concentration of $10^{7} \mathrm{CFU} / \mathrm{mL}$ were spread on the PLLA and Ag/PLLA membranes, respectively, before being incubated at $37^{\circ} \mathrm{C}$ for 2 hours. SEM observations were then performed.

As shown in Figure 4, the number of Staph. spread on PLLA membrane was obviously more than that on Ag/PLLA membrane. Besides, the surface of Staph. on PLLA membrane was smoother than that on Ag/PLLA membrane. This result indicated that there might be less destroy on the structure of bacteria which spread on PLLA membrane than that on Ag/PLLA membrane.

\section{Discussion}

Tissue engineering scaffolds with the effectiveness in reducing the possibility of infections will be more acceptable for medical applications [13]. For example, skin is the first protective barrier for all the tissues and organs, so a wound on skin will easily weaken the anti-infective capability of human body. Here we mainly focused on the feasibility to fabricate an antibacterial scaffolds for wound healing.

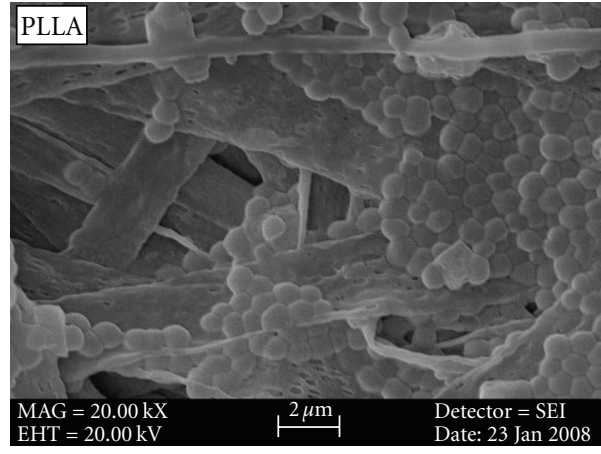

(a)

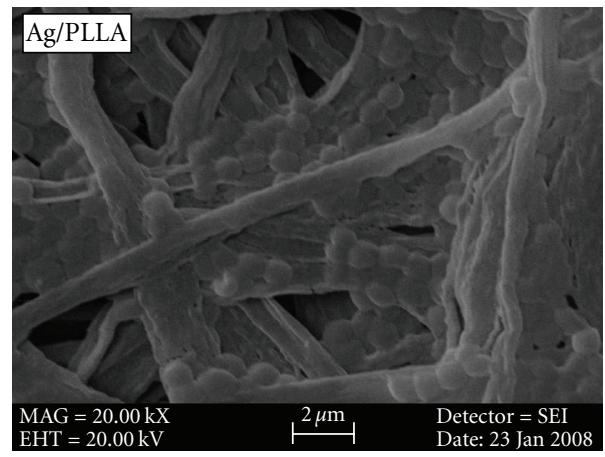

(b)

FIgure 4: SEM images magnified Staph. on PLLA and Ag/PLLA fibrous membrane 20 thousand times $(20 \mathrm{kV})$. (1) The number of Staph. on PLLA membranes was more than that on the Ag/PLLA membrane. (2) Staph. on PLLA membrane had smooth surface, while the ones on Ag/PLLA membrane were not very smooth.

Nanosilver was observed to be toxic to bacteria cells. This finding suggested an exploration of the antibacterial tissue engineering scaffolds $[12,13,24]$. In 2004, Alt et al. reported that bone cement with $1 \%$ of nanosilver showed high antibacterial activity against most of the tested bacteria [13]. Yeo et al. found that fibers having nanosilver showed excellent antibacterial property. Besides, PLLA was already approved by FDA as a safe material for the clinical application. Thus, to combine silver nanoparticles and PLLA by using electrospinning technique [1-4] could be a good approach to produce tissue engineering scaffolds with high antibacterial effect. On the other hand, electrospun fibers can produce ventilate fibrous membranes with wide interface to highly perform their antibacterial activity.

In the antibacterial tests, PLLA membranes were set as control specimen. The Ag/PLLA membranes were found to inhibit the proliferation of E. coli and Staphylococcus significantly. On the basis of this finding, silver nanoparticles were approved to be released from PLLA fibers gradually. In terms of the degradability of PLLA, Ag/PLLA is an optimal matrix to contain silver nanoparticles and help to gradually release silver nanoparticles as PLLA degrade. Accordingly, $\mathrm{Ag} / \mathrm{PLLA}$ fibrous membrane is a good candidate antibacterial substance for tissue engineering. 
TABLE 1: Assessment of the antibacterial activity of the extractions of Ag/PLLA membranes.

\begin{tabular}{|c|c|c|}
\hline \multirow{2}{*}{ Bacteria } & \multicolumn{2}{|c|}{ Average width of bacteria inhibition clear zone (mm) } \\
\hline & PLLA & $5 \% \mathrm{Ag} / \mathrm{PLLA}$ \\
\hline \multicolumn{3}{|l|}{ Before } \\
\hline Escherichia coli & 0 & $5.0 \pm 0.1^{*}$ \\
\hline Staphylococcus aureus & 0 & $5.0 \pm 0.1^{*}$ \\
\hline \multicolumn{3}{|l|}{ After } \\
\hline Escherichia coli & 0 & $1.2 \pm 0.1^{*}$ \\
\hline Staphylococcus aureus & 0 & $1.5 \pm 0.1^{*}$ \\
\hline
\end{tabular}

${ }^{*}$ The mean values were shown for means \pm SD. $P<.05$ versus PLLA membranes.

Meanwhile, although the Ag/PLLA membrane was proved to be antibacterial in this study, the antibacterial activity of the membrane was observed decreasing. After eight-week exposure in the air, the antibacterial activity of the Ag/PLLA fibrous membranes reduced. This result may be caused by the oxidization by the air or other reactions during the exposure. So, further investigation about the factors which affect the antibacterial properties of Ag/PLLA membrane need to be performed in the next process. These findings also suggest that it is possible to keep Ag/PLLA membrane with strong antibacterial activity for long time.

To investigate the mechanism of the antibacterial activity of Ag/PLLA membranes, morphological change of bacteria were primarily observed by SEM. More Staph. were seen on PLLA membrane than that on Ag/PLLA membrane. And the surface of Staph. on PLLA membranes was smoother than that on Ag/PLLA membranes. These findings imply that nanosilver may affect the structure of bacterial membrane, thereby cause the defunctionalization of bacteria. The mechanism of antibacterial activity of Ag/PLLA membranes needs further study to be verified.

On the other hand, based on the review of silver toxicity studies, it was reported that the possible side effects of nanoparticles had not been much investigated, so, detailed study needs to be carried out before the merchandised product is introduced to the market [30]. But, as a strong antibacterial substance, silver nanoparticles have been playing an irreplaceable role in anti-infections. Hence, the further study needs to be carried on for both the antibacterial function and toxicity of silver nanoparticles composed materials to find out the safe balance point for antibacterial scaffolds with no toxicity to the human body and environment.

\section{Conclusions}

This study describes the antibacterial capability of the Ag/PLLA fibrous membrane. On the basis of the observations and findings above, it can be concluded that PLLA is a good matrix for containing and gradually releasing antibacterial substance, while silver nanoparticles can provide strong antibacterial property to the Ag/PLLA fibrous membranes. Thus, Ag/PLLA fibrous membrane can be used as an antibacterial scaffold for wound healing. Besides, the factors which influence the antibacterial effect need further investigation to clarify the mechanism of antibacterial activity of Ag/PLLA membranes.

\section{Acknowledgments}

The authors would like to express their gratitude to the Hong Kong Research Grant Council, the Technology Innovation Commission/HKRITA, and the Hong Kong Polytechnic University for supporting this research through Projects PolyU 5281/03E, ITP-001-07-TP, and G-YE51.

\section{References}

[1] S. Sukigara, M. Gandhi, J. Ayutsede, M. Micklus, and F. Ko, "Regeneration of Bombyx mori silk by electrospinning-part 1: processing parameters and geometric properties," Polymer, vol. 44, no. 19, pp. 5721-5727, 2003.

[2] Y. Chen, A. F. T. Mak, M. Wang, J. Li, and M. S. Wong, "PLLA scaffolds with biomimetic apatite coating and biomimetic apatite/collagen composite coating to enhance osteoblastlike cells attachment and activity," Surface and Coatings Technology, vol. 201, no. 3-4, pp. 575-580, 2006.

[3] K. E. Park, H. K. Kang, S. J. Lee, B. M. Min, and W. H. Park, "Biomimetic nanofibrous scaffolds: preparation and characterization of PGA/Chitin blend nanofibers," Biomacromolecules, vol. 7, no. 2, pp. 635-643, 2006.

[4] S. Sukigara, M. Gandhi, J. Ayutsede, M. Micklus, and F. Ko, "Regeneration of Bombyx mori silk by electrospinningpart 2: process optimization and empirical modeling using response surface methodology," Polymer, vol. 45, no. 11, pp. 3701-3708, 2004.

[5] S. Putthanarat, R. K. Eby, W. Kataphinan, et al., "Electrospun Bombyx mori gland silk," Polymer, vol. 47, no. 15, pp. 56305632, 2006.

[6] H. K. Noh, S. W. Lee, J.-M. Kim, et al., "Electrospinning of chitin nanofibers: degradation behavior and cellular response to normal human keratinocytes and fibroblasts," Biomaterials, vol. 27, no. 21, pp. 3934-3944, 2006.

[7] K. Tomihata and Y. Ikada, "In vitro and in vivo degradation of films of chitin and its deacetylated derivatives," Biomaterials, vol. 18, no. 7, pp. 567-575, 1997.

[8] W.-J. Li, C. T. Laurencin, E. J. Caterson, R. S. Tuan, and F. K. Ko, "Electrospun nanofibrous structure: a novel scaffold for tissue engineering," Journal of Biomedical Materials Research, vol. 60, no. 4, pp. 613-621, 2002. 
[9] W.-J. Li, R. Tuli, X. Huang, P. Laquerriere, and R. S. Tuan, "Multilineage differentiation of human mesenchymal stem cells in a three-dimensional nanofibrous scaffold," Biomaterials, vol. 26, no. 25, pp. 5158-5166, 2005.

[10] Y. Ji, K. Ghosh, X. Z. Shu, et al., "Electrospun threedimensional hyaluronic acid nanofibrous scaffolds," Biomaterials, vol. 27, no. 20, pp. 3782-3792, 2006.

[11] D. H. Reneker and I. Chun, "Nanometre diameter fibres of polymer, produced by electrospinning," Nanotechnology, vol. 7, no. 3, pp. 216-223, 1996.

[12] M. Li, M. J. Mondrinos, M. R. Gandhi, F. K. Ko, A. S. Weiss, and P. I. Lelkes, "Electrospun protein fibers as matrices for tissue engineering," Biomaterials, vol. 26, no. 30, pp. 59996008, 2005.

[13] V. J. Chen and P. X. Ma, "The effect of surface area on the degradation rate of nano-fibrous poly(L-lactic acid) foams," Biomaterials, vol. 27, no. 20, pp. 3708-3715, 2006.

[14] X. Chen and H. J. Schluesener, "Nanosilver: a nanoproduct in medical application," Toxicology Letters, vol. 176, no. 1, pp. 112, 2008.

[15] V. Alt, T. Bechert, P. Steinrücke, et al., "An in vitro assessment of the antibacterial properties and cytotoxicity of nanoparticulate silver bone cement," Biomaterials, vol. 25 , no. 18, pp. 4383-4391, 2004.

[16] B. Shan, Y.-Z. Cai, J. D. Brooks, and H. Corke, "Antibacterial properties of Polygonum cuspidatum roots and their major bioactive constituents," Food Chemistry, vol. 109, no. 3, pp. 530-537, 2008.

[17] Y. Li, P. Leung, L. Yao, Q. W. Song, and E. Newton, "Antimicrobial effect of surgical masks coated with nanoparticles," Journal of Hospital Infection, vol. 62, no. 1, pp. 58-63, 2006.

[18] T. A. Gaonkar, L. A. Sampath, and S. M. Modak, "Evaluation of the antimicrobial efficacy of urinary catheters impregnated with antiseptics in an in vitro urinary tract model," Infection Control and Hospital Epidemiology, vol. 24, no. 7, pp. 506-513, 2003.

[19] B. Walder, D. Pittet, and M. R. Tramèr, "Prevention of bloodstream infections with central venous catheters treated with anti-infective agents depends on catheter type and insertion time: evidence from a meta-analysis," Infection Control o Hospital Epidemiology, vol. 23, no. 12, pp. 748-756, 2002.

[20] N. S. Morris and D. J. Stickler, "Encrustation of indwelling urethral catheters by Proteus mirabilis biofilms growing in human urine," Journal of Hospital Infection, vol. 39, no. 3, pp. 227-234, 1998.

[21] B. Galeano, E. Korff, and W. L. Nicholson, "Inactivation of vegetative cells, but not spores, of Bacillus anthracis, $B$. cereus, and B. subtilis on stainless steel surfaces coated with an antimicrobial silver- and zinc-containing zeolite formulation," Applied and Environmental Microbiology, vol. 69, no. 7, pp. 4329-4331, 2003.

[22] V. Thomas, M. M. Yallapu, B. Sreedhar, and S. K. Bajpai, "A versatile strategy to fabricate hydrogel-silver nanocomposites and investigation of their antimicrobial activity," Journal of Colloid and Interface Science, vol. 315, no. 1, pp. 389-395, 2007.

[23] K. K. Lai and S. A. Fontecchio, "Use of silver-hydrogel urinary catheters on the incidence of catheter-associated urinary tract infections in hospitalized patients," American Journal of Infection Control, vol. 30, no. 4, pp. 221-225, 2002.

[24] J. H. Crabtree, R. J. Burchette, R. A. Siddiqi, I. T. Huen, L. L. Hadnott, and A. Fishman, "The efficacy of silver-ion implanted catheters in reducing peritoneal dialysis-related infections," Peritoneal Dialysis International, vol. 23, no. 4, pp. 368-374, 2003.
[25] D. K. Riley, D. C. Classen, L. E. Stevens, and J. P. Burke, "A large randomized clinical trial of a silver-impregnated urinary catheter: lack of efficacy and staphylococcal superinfection," The American Journal of Medicine, vol. 98, no. 4, pp. 349-356, 1995.

[26] F. Furno, K. S. Morley, B. Wong, et al., "Silver nanoparticles and polymeric medical devices: a new approach to prevention of infection?" Journal of Antimicrobial Chemotherapy, vol. 54, no. 6, pp. 1019-1024, 2004.

[27] M. Wilson, R. McNab, and B. Henderson, Bacterial Disease Mechanisms: An Introduction to Cellular Microbiology, Cambridge University Press, Cambridge, UK, 2002.

[28] S. Y. Yeo, H. J. Lee, and S. H. Jeong, "Preparation of nanocomposite fibers for permanent antibacterial effect," Journal of Materials Science, vol. 38, no. 10, pp. 2143-2147, 2003.

[29] J. Li, Y. Li, L. Li, A. F. T. Mak, F. Ko, and L. Qin, "Fabrication of poly(L-latic acid) scaffolds with wool keratin for osteoblast cultivation," Advanced Materials Research, vol. 47-50, pp. 845848, 2008.

[30] M. Rai, A. Yadav, and A. Gade, "Silver nanoparticles as a new generation of antimicrobials," Biotechnology Advances, vol. 27, no. 1, pp. 76-83, 2009. 

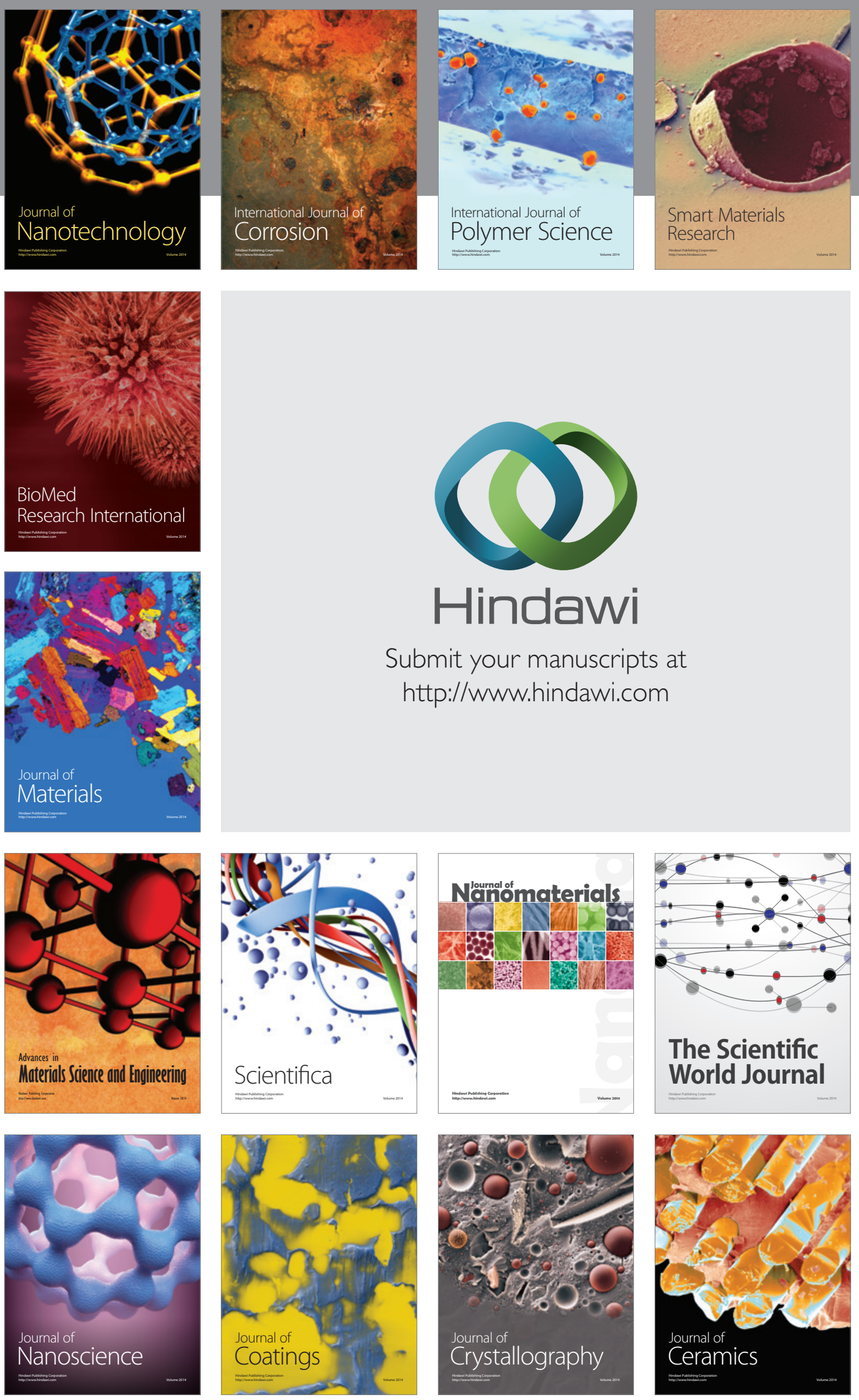

The Scientific World Journal

Submit your manuscripts at

http://www.hindawi.com

\section{World Journal}

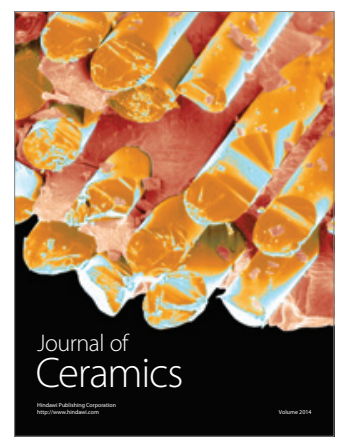

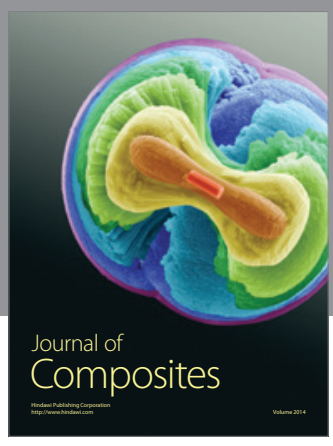
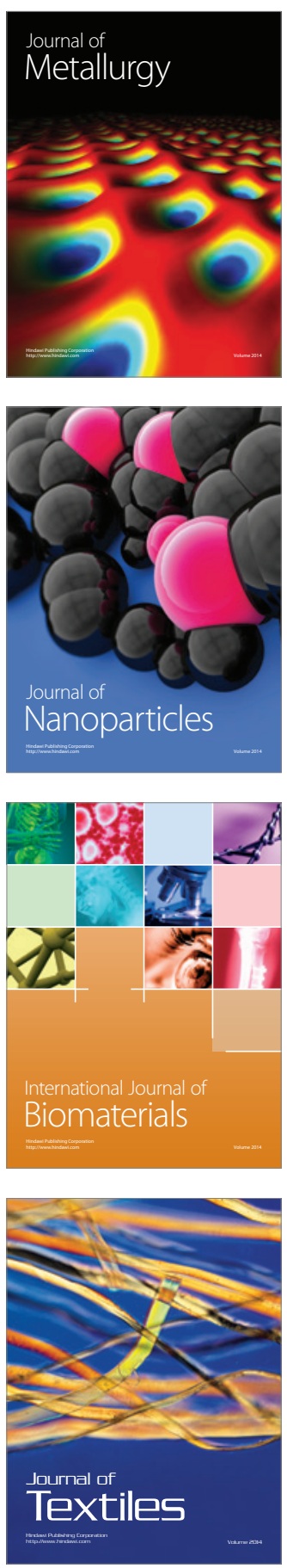